\title{
ASSOCIAÇÕES ENTRE CARACTERÍSTICAS ANDROLÓGICASE DE CARCAÇA EM TOUROS NELORE
}

\author{
ASSOCIATIONS BETWEEN ANDROLOGY AND QUALITY OF CARCASS TRAITS IN \\ NELLORE BULLS \\ Lopes, D.T. ${ }^{1 *}$; Viu, M.A.O. ${ }^{1 A}$; Ferraz, H.T. ${ }^{1 \mathrm{~B}}$; Mascioli, A.S. ${ }^{1 \mathrm{C}}$; Viu, A.F.M. ${ }^{1 \mathrm{D}}$; Dias, L.R.O. ${ }^{1 \mathrm{E}}$; \\ Santos, K.J.G. ${ }^{2}$ e Magnabosco, C.U. ${ }^{3}$

\begin{abstract}
1Universidade Federal de Goiás. Campus Jataí (CAJ/UFG). Brasil. *dtl.ufg@gmail.com; Amarcoviu@yahoo.com.br; Bhtferraz@gmail.com; Camascioli7@hotmail.com; Daleviuufg@yahoo.com.br; Elr_dias@hotmail.com

${ }^{2}$ Universidade Estadual de Goiás. São Luís dos Montes Belos-GO. Brasil. klaytovet@yahoo.com.br ${ }^{3}$ Embrapa Cerrados. Planaltina-DF. Brasil. mclaudio@cnpaf.embrapa.br
\end{abstract}

\section{Palavras chave adicionaIS}

Correlação genética. Qualidade seminal.Zebuínos.

\section{RESUMO}

Objetivou-se com este estudo verificar a existência de associações genéticas entre características de carcaça e características andrológicas em touros Nelore jovens. Realizouse avaliação andrológica e da carcaça de 223 machos Nelore com média de 21 meses de idade. Para estimação dos parâmetros genéticos utilizouse a inferência bayesiana por meio do software THRGIBBS1F90. As estimativas de herdabilidade obtidas para DEFMAI, DEFMEN, DEFTOT, AR, AOL, EG e P8 foram 0,$33 ; 0,20 ; 0,21 ; 0,35 ; 0,32 ; 0,28$ e 0,29 , respectivamente. As estimativas de correlação genética de DEFTOT com AOL, EG e P8 foram $-0,46 ;-0,28$ e $-0,19$, respectivamente; já de AR com estas mesmas características foram $-0,60 ;-0,03$ e $-0,12$, respectivamente. Os resultados obtidos neste estudo demonstram haver resposta correlacionada favorável entre as características reprodutivas e de carcaça estudadas.

\section{SUMMARY}

The aim with this study was to verify the existence of genetic associations among carcass traits, and andrology traits in Nellore young bulls. Breeding soundness and carcass examinations were done on 223 Nellore males with average of 21 months age. To estimate the genetic parameters the bayesian inference was used through the

\author{
AdDITIONAL KEYWORDS \\ Genetic correlation. Seminal quality. Zebu cattle.
}

software THRGIBBS1F90. The heritability estimates was obtained for DEFMAI, DEFMEN, DEFTOT, AR, AOL, EG and P8 were 0.33, 0.20, $0.21,0.35,0.32,0.28$, and 0.29 , respectively. The genetic correlation estimates of DEFTOT with $\mathrm{AOL}, \mathrm{EG}$ and $\mathrm{P} 8$ were $-0.46,-0.28$, and -0.19 , respectively; to $A R$ with these same characteristics were $-0.60,-0.03$, and -0.12 , respectively. The results of this study demonstrate that there is a favorable correlated response between reproductive and carcass traits studied.

\section{INTRODUÇÃO}

Sabe-se que a reprodução é um dos fatores que mais afetam a produtividade do rebanho. Nesse contexto, os aspectos reprodutivos dos machos, entre eles a qualidade seminal, têm ainda mais importância, visto que cada reprodutor transfere $50 \%$ do seu material genético a um número muito grande de matrizes. A melhor maneira de conhecer a qualidade seminal é por meio da realização de avaliação andrológica completa, que apesar de sua importância, constitui prática pouco utilizada pelos pecuaristas (Viu, 2009).

Ao se tratar de melhoria de qualidade da 
carne, tem-se hoje em mãos uma ferramenta muito útil: a ultrassonografia de carcaça. Essa tecnologia permite estimar, com precisão, em animais vivos, a composição da carcaça, possibilitando a estimação do mérito genético para qualidade de carcaça. Isto permite a seleção e obtenção de produtos comercialmente mais desejáveis (Tarouco, 2004).

Diante do exposto, fica evidente a necessidade de selecionar animais para precocidade sexual e também para qualidade de carcaça.

Objetivou-se com este trabalho verificar a existência de associações genéticas entre características de carcaça, obtidas in vivo por ultrassonografia, e características reprodutivas obtidas por meio de avaliações andrológicas em touros Nelore jovens.

\section{MATERIALE MÉTODOS}

Neste estudo foram utilizadas 223 avaliações andrológicas e de carcaça de touros jovens da raça Nelore, com idade média de 21 meses, criados em sistemas de produção a pasto na Fazenda Capivara, do Centro Nacional de Pesquisa em Arroz e Feijão, CNPAF/EMBRAPA. Foram avaliadas as características: defeitos espermáticos maiores (DEFMAI); defeitos espermáticos menores (DEFMEN); defeitos espermáticos totais (DEFTOT); aptidão reprodutiva (AR); área de olho de lombo (AOL); espessura de gordura subcutânea (EG) e espessura de gordura subcutânea na garupa (P8).

Os touros foram submetidos a exame clínico geral e do sistema reprodutor de acordo com as recomendações do Colégio Brasileiro de Reprodução Animal (CBRA, 1998).

Com base nas informações obtidas determinaram-se as categorias para a característica AR, sendo: sexualmente imaturos (motilidade $<50 \%$, vigor $<3$ ou defeitos totais $>30 \%$, desde que fosse detectada alta contagem de gotas citoplasmáticas proximais); ou aptos à reprodução (motilidade $\geq 50 \%$; vigor $\geq 3$ e defeitos totais $<30 \%$ ).

As características de carcaça foram avaliadas conforme descrito por Dibiasi (2006).

Na estimação dos parâmetros genéticos, utilizou-se a inferência bayesiana por meio do software THRGIBBS1F90 (Tsuruta e Miztal, 2006), sob modelo animal. Para as características estudadas considerou-se como efeitos fixos o grupo de contemporâneos e o ano de coleta, além da idade do animal no momento da coleta como covariável. O modelo completo pode ser representado em notação matricial como:

$$
y=X \beta+Z_{1} a+e
$$

em que:

$y=$ é o vetor das observações (características andrológicas e de carcaça);

$\beta=$ é o vetor dos efeitos fixos (data da coleta e grupo contemporâneo, além da covariável idade do animal no momento da coleta);

$a=$ é o vetor dos efeitos aleatórios que representam os valores genéticos aditivos direto de cada animal;

$e=0$ vetor de efeitos aleatórios residuais;

$X, Z_{1}=$ são as matrizes de incidência que relacionam as observações aos efeitos fixos, aos efeitos aleatórios genéticos aditivos direto e aos efeitos aleatórios residuais, respectivamente. Oarquivo de genealogia incluiu 1434 animais da raça Nelore.

As características foram analisadas de forma univariada com intuito de obter as estimativas de componentes de (co)variância e, posteriormente, de forma bivariada, obtendo-se assim as variâncias e covariâncias, as correlações genéticas aditivas e residuais e as herdabilidades das características em estudo.

No modelo de limiar assume-se que a escala subjacente apresenta distribuição normal.

Para as análises bivariadas, envolvendo categóricas e contínuas de acordo com o enfoque bayesiano, admitiu-se que as distribuições iniciais dos efeitos aleatórios 
genéticos e residuais seguem distribuição normal multivariada.

Na implementação da Amostragem de Gibbs foi utilizado tamanho de cadeia inicial de 1000000 de ciclos, sendo que os primeiros 300000 ciclos foram descartados e as amostras retiradas a cada 1000 ciclos, totalizando 700 amostras iniciais. As análises das amostras, da correlação serial e da convergência da cadeia de Gibbs foram realizadas com o auxílio do programa GIBANAL (Van Kaam, 1998).

O parâmetro $v$ é o grau de liberdade correspondente à distribuição Wishart invertida, indicando o grau de confiabilidade da distribuição inicial. Neste estudo, o parâmetro $v$ utilizado foi de valor zero, ou seja, não refletiu nenhum grau de conhecimento sobre os parâmetros.

O erro de Monte Carlo, de grande utilidade para garantir a convergência das cadeias de Monte Carlo, foi estimado calculando a raiz da variância das amostras retiradas para cada componente de (co)variância e dividindo esta variância pelo número de amostras.

\section{RESULTADOSEDISCUSSÃO}

As correlações genéticas e as covariân- cias genéticas e residuais entre as características estudadas estão sumarizadas na tabela I.

Nota-se que as estimativas de correlação genética entre as características relacionadas à qualidade da carcaça, medidas in vivo por ultrassonografia (AOL, EG e P8), e aquelas relacionadas à qualidade seminal dos touros (DEFMAI, DEFMEN e DEFTOT) foram todas negativas, podendo ser consideradas de moderada magnitude. Essa informação é de grande valia, pois indica que é possível, ao selecionar animais para qualidade de carcaça, obter melhoria também na função reprodutiva, expressa pela melhor qualidade do ejaculado. Esse comportamento entre as características EG e P8 com a porcentagem de defeitos espermáticos já era esperado, pois de acordo com Lehninger et al. (1995), o colesterol, um tipo de lipídio, é precursor da testosterona, andrógeno que estimula o crescimento testicular e a produção de espermatozoides, justificando a melhoria da qualidade do ejaculado quando há aumento da EG e da P8. As estimativas de correlação mais expressivas foram obtidas entre AOL e as características reprodutivas, ficando claro que quanto maior a $\mathrm{AOL}$, menor a quantidade de

Tabela I. Correlações genéticas aditivas ( rg) e erro padrão de Monte Carlo (EPMC) entre as características relacionadas à qualidade seminal e de carcaça de touros jovens da raça Nelore, obtidas a partir de análises bayesianas bivariadas sob modelo animal. (Additive genetic correlations and standard error of Monte Carlo (EPMC) between traits related to sperm quality and carcass of young Nellore bulls, obtained from bivariate Bayesian analyzes by animal model).

\begin{tabular}{lcccccr}
\hline Variáveis & \multicolumn{2}{c}{ AOL } & \multicolumn{2}{c}{ EG } & \multicolumn{2}{c}{ P8 } \\
& rg & EPMC & rg & EPMC & rg & EPMC \\
\hline DEFMAI & $-0,18$ & 0,0089 & $-0,09$ & 0,0082 & $-0,06$ & 0,0084 \\
DEFMEN & $-0,65$ & 0,0063 & $-0,57$ & 0,0081 & $-0,38$ & 0,0079 \\
DEFTOT & $-0,46$ & 0,0082 & $-0,28$ & 0,0041 & $-0,19$ & 0,0073 \\
AR & 0,60 & 0,0085 & 0,03 & 0,0082 & 0,12 & 0,0083 \\
\hline
\end{tabular}

$\mathrm{AOL}=$ área de olho de lombo; $\mathrm{EG}=$ espessura de gordura subcutânea; P8= espessura de gordura subcutânea na garupa; $D E F M A I=$ defeitos espermáticos maiores; DEFMEN= defeitos espermáticos menores; DEFTOT= defeitos espermáticos totais; $A R=$ aptidão reprodutiva. 
defeitos espermáticos e melhor a AR, ou seja, animais aptos à reprodução.

Com relação à AR, esta apresentou correlação genética positiva com todas as característica relacionadas à qualidade da carcaça, sendo esta de moderada a alta magnitude com a AOL $(0,60)$ e de baixa magnitude com EG $(0,03)$ e P8 $(0,12)$. Essa informação auxilia muito os melhoristas, pois significa que ao selecionar para AOL obtémse, como resposta correlacionada, melhoria da AR. Já ao selecionar para EG e P8 essa resposta correlacionada é irrisória mas favorável, não prejudicando a fertilidade. Infelizmente não foi possível contrastar esses resultados com outros, visto que na literatura consultada não foram encontrados trabalhos que seguiram essa mesma linha de raciocínio. Esse fato, inclusive, demonstra a importância das informações prestadas como também a necessidade de estudos mais aprofundados sobre tema.

$\mathrm{Na}$ verdade foi possível perceber que, tanto as características andrológicas quanto

\section{BIBLIOGRAFIA}

CBRA. 1998. Colégio Brasileiro de Reprodução. Manual para exame andrológico e avaliação de sêmen animal. 2․ . ed. Belo Horizonte. 49 pp.

Dibiasi, N.F. 2006. Estudo do crescimento, avaliação visual, medidas por ultrassonografia e precocidade sexual em touros jovens pertencentes a 21 raças com aptidão para corte. Tese de Doutorado. UNESP. 94 pp.

Lehninger, A.L.; Nelson, D.L. e Cox, M.M. 1995. Princípios de bioquímica. Sarvie. São Paulo. 839 pp.

Tarouco, J. 2004. A história do ultra-som no Brasil. 1 ํ Workshop de ultrassonografia para avaliação de carcaça bovina. Pirassununga-SP.

Tsuruta, S. e Miztal, I. 2006. THRGIBBS1F90 for as de carcaça avaliadas, possuem grande influência ambiental, demonstrada pelas baixas estimativas de herdabilidade. Isso quer dizer que quando há boas condições ambientais, principalmente oferta de forragem, os animais apresentarão maiores medidas de carcaça e também menor quantidade de defeitos espermáticos.

\section{CONCLUSÕES}

Os resultados deste estudo demonstraram haver possibilidade de melhorar a qualidade seminal de touros Nelore jovens concomitantemente à melhoria da qualidade da carcaça, considerando as estimativas de correlação genética obtidas.

\section{AGRADECIMENTOS}

À Coordenação de Aperfeiçoamento de Pessoal de Nível Superior (CAPES) e à Empresa Brasileira de Pesquisa Agropecuária (EMBRAPA), por terem acreditado e fomentado este estudo.

estimation of variance components with threshold and linear models. Proc. $8^{\text {th }}$ World Congress on Genetics Applied to Livestock Production. Belo Horizonte. Minas Gerais, Brazil, pp. 27-31

Van Kaam, J.B.C.H.M. 1998. Gibanal2.9: Analyzing program for Markov chain Monte Carlo sequences. Dep. Anim. Sci., Wageningen Agricultural University. Wageningen. The Netherlands.

Viu, M.A.O. 2009. Estudo genético quantitativo e ambiental do potencial reprodutivo de touros Nelore criados no Centro-Oeste do Brasil. Tese de Doutorado. Universidade Federal de Goiás. UFG. 118 pp. 